\title{
Reliable detection of abnormal ozone measurements using an air quality sensors network
}

\author{
Fouzi Harrou, Member, IEEE, Abdelkader Dairi, Ying Sun, Mohamed Senouci
}

\begin{abstract}
Ozone pollution is one of the most important pollutants that have a negative effect on human health and the ecosystem. An effective statistical methodology to detect abnormal ozone measurements is proposed in this study. We used a Deep Belief Network model to account for nonlinear variation of ground-level ozone concentrations, in combination with a one-class support vector machine, for detecting abnormal ozone measurement. We assessed the efficiency of this methodology by using real data from a network of air quality monitoring systems in Isère, France. Results demonstrated the capability of the proposed strategy to identify abnormalities in ozone measurements.
\end{abstract}

Index Terms-Ozone pollution, machine learning, statistical monitoring, anomaly detection.

\section{INTRODUCTION}

Pollution by tropospheric ozone is an increasing problem in industrialized countries [1]. Both ambient pollutants and greenhouse gases come from three major sources: transport, heating, and industry. Beyond these common sources, climate change and air pollution also have intertwined effects. The high concentrations of air pollution continue to have a significant impact on the health of Europeans ${ }^{1}$. Ozone pollution is one of the most important pollutants that have a negative effect on human health and the ecosystem [2], [3]. Ozone is the reactive form of oxygen that contains three atoms per molecule $\left(\mathrm{O}_{3}\right)$ rather than the normal two. Ground-level ozone is created by chemical reactions between other pollutants, especially oxides of nitrogen and carbon-based compounds released in vehicle exhausts and by many industrial processes [2], [3]. The reactions that create ozone are promoted by bright sunlight, often leading to the serious air quality problem called photochemical smog. Ozone is a troublesome pollutant at ground level and throughout the lower layer of the atmosphere [4], [5]. Exposure to the ozone can cause breathing difficulties, eye irritation and other health problems, and may also harm crops and other vegetation [6], [7]. Air quality monitoring and management are primordial to ensuring a suitable environment for health and a quality living environment [8]. Thus, detecting abnormal ozone levels is essential to improving air quality management and take the proper precautions. This will allow us to warn the public of harmful ozone levels, but also to check the sensors in case of a technical problem is the reason for an

F. Harrou and Y. Sun are with King Abdullah University of Science and Technology (KAUST) Computer, Electrical and Mathematical Sciences and Engineering (CEMSE) Division, Thuwal, 23955-6900, Saudi Arabia

A. Dairi and M. Senouci are with Computer Science Department, University of Oran 1 Ahmed Ben Bella, Street El senia el mnouer bp 31000 Oran, Algeria

${ }^{1}$ Air quality in Europe 2017, Rapport, Agence Européenne de l'Environnement, October 2017 anomaly. The objective of this paper is to propose an automatic monitoring approach to detect abnormal ozone measurements in a regional ozone monitoring network.

In recent years, there has been much discussion on deep learning-based feature extraction in the literature [9], [10], [11]. Indeed, deep learning-based methods have been developed for modeling complex processes with flexibility, simplicity, and strength. They have been successfully applied to modeling complex process using series of multilayer architectures. They have been widely used for many applications including traffic flow prediction [12], traffic speed prediction [9], obstacle detection for autonomous vehicles [13], health informatics [10], monitoring of water resource recovery facilities [14], learning spatiotemporal features from tomography sensors [11], localization activity, and gesture recognition [15] and in fingerprints indoor positioning based on WIFI [16]. Till now, however, the Deep Belief Networks (DBNs) model has not been considered in air quality monitoring.

This study aims to develop a deep learning-based approach that can properly detect ozone anomalies. Specifically, the proposed approach integrates a DBN modeling approach and one-class support vector machine (OCSVM). One benefit with the proposed detection system is that both advantages of the powerful feature extraction capability of DBNs and superior predicting capacity of OCSVM can be exploited. This approach has been evaluated using real ozone data from Isére region, France. The following section briefly reviews the DBN model and OCSVM classifier. Section III introduces the proposed DBN-based OCSVM algorithm. Section IV investigates the effectiveness of the proposed approach in detecting abnormal ozone measurements using real dataset. Section V lists the conclusions of the study.

\section{PRELIMINARY MATERIAL}

\section{A. Deep Belief Networks (DBNs)}

DBNs are designed based on stacking succession of Restricted Boltzmann Machines (see Figure 1), which are defined as a stochastic neural network [17]. Boltzmann machines (BM) are undirected graphical models, which are also considered as Markov Random Fields [18]. They are a powerful tool for estimating a probability distribution of input datasets. Restricted Boltzmann Machines (RBMs) are a special case of BMs, interpreted as stochastic neural networks and characterized to be energy-based models.

DBNs are graphical models (see figure 2), trained in an unsupervised way with unlabeled data. They are applied to 


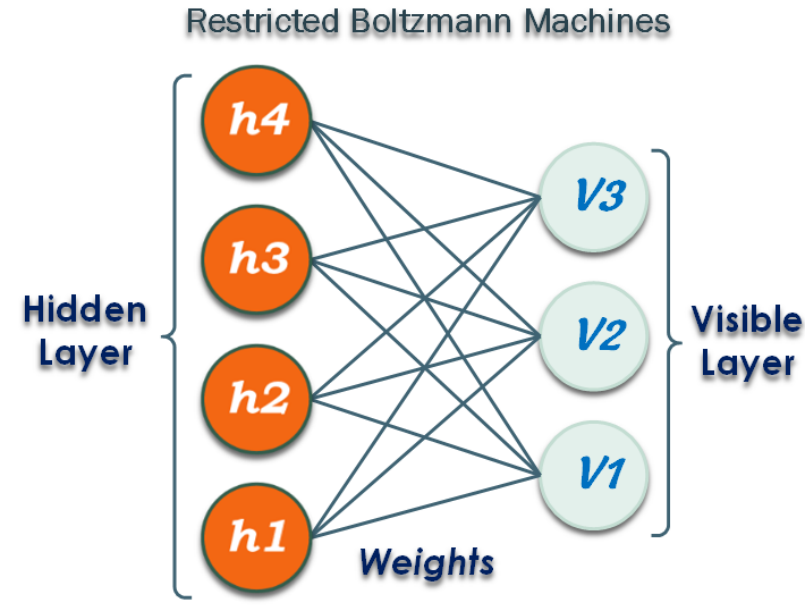

Fig. 1. RBM structure

extract and discover a complex hierarchical representation of the training data through multiple transformations of inputs over layers.

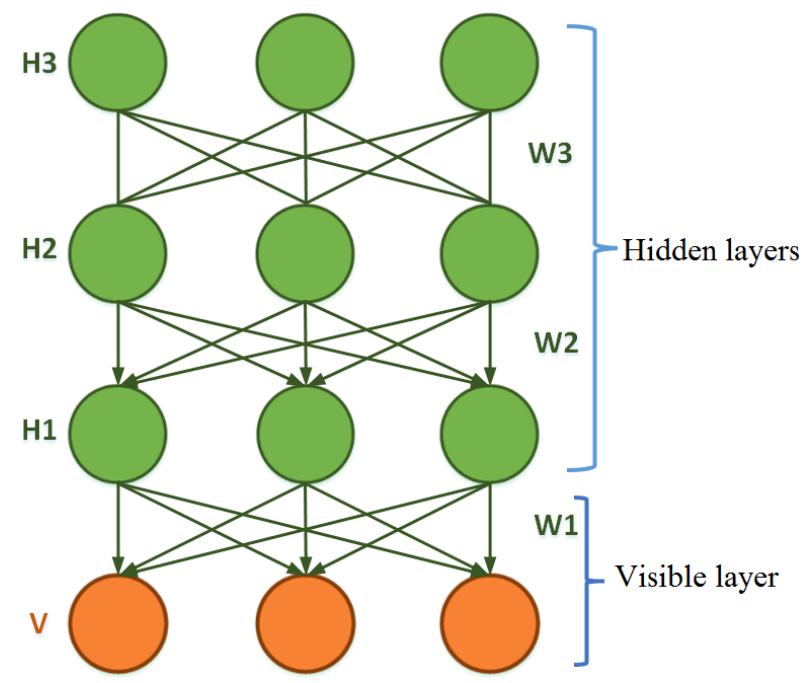

Fig. 2. A diagrammatic illustration of Deep belief networks, where $W 1, W 2$ and $W 3$ represent respectively weight matrix of RBM1 (Layer 1), RBM2 (Layer 2) and RBM3 (Layer 3); they are computed in the layer-wise greedy training.

The key idea of the training method introduced by [19] is to train the deep network Greedy layer-wise (sequentially), where each stacked RBM is trained separately using unlabeled dataset (unsupervised). After the training phase, each $k$-th RBM has its own weight matrix $W_{k}$ as depicted in Figure 1. The neural network topology [19] is constructed based on both undirected model (RBM) as output and Sigmoid Belief Networks (directed) composed of ell hidden layers.

The joint distribution of stacked RBMs that compose a DBN could model the interaction linking observed vector $x$ and $\ell$ hidden layers $h$ (see Figure 1) as follow:

$$
\begin{aligned}
P\left(x, h^{1}, h^{2}, \ldots, h^{l}\right)= & \left(P\left(x \mid h^{1}\right) P\left(h^{1} \mid h^{2}\right) \ldots P\left(h^{l-2} \mid h^{l-1}\right)\right) \\
& P\left(h^{\ell-1}, h^{\ell}\right),
\end{aligned}
$$

where $P\left(x \mid h^{1}\right)$ term denote conditional distribution of the input $x$ and the first hidden layer $h^{1}\left(h^{1} \Rightarrow x\right), P\left(h^{1} \mid h^{2}\right)$ model the conditional distribution of hidden layer and each other as Sigmoid Belief Networks (directed graph see Figure 1) $h^{2} \Rightarrow h^{1}$. The term $P\left(h^{\ell-1}, h^{\ell}\right)$ express the joint distribution of an RBM. We can write the DBN probabilistic model in simple form:

$$
P\left(x, h^{1}, \ldots, h^{l}\right)=\left(\prod_{k=0}^{\ell-2} P\left(h^{k} \mid h^{k+1}\right)\right) P\left(h^{\ell-1}, h^{\ell}\right),
$$

where $x=h^{0}$ and $P\left(h^{k} \mid h^{k+1}\right)=\operatorname{sigmoid}\left(b^{k}+W^{k} h^{k+1}\right)$ represents a visible given hidden conditional distribution in an RBM corresponsig to level $k$ of the DBN and $P\left(h^{\ell-1}, h^{\ell}\right)$ represents the joint distribution in the top-level RBM.

\section{B. The one-class SVM (OCSVM):}

OSVM [20] scheme is applied to solve one class classification problems and deal with high dimensionality case. OCSVM is trained in unsupervised fashion based on an unlabeled normal data to construct boundaries also known as hyperplanes. The determined boundaries are used to evaluate new testing observations if they share similar features as the normal class in features space $\mathcal{F}$ for normal observation. Setting up OCSVM boundaries allow to assess the membership or not of newly observed data to the same features space of training dataset delimited by hyperplanes. This assessment is done via a decision function. The decision function response is -1 for outliers and +1 for inliers. The decision function $f(x)$ maximizes the distance between the hyperplane $\mathcal{H}$ separating the training dataset in $\mathcal{F}$ features space and the origin (see Figure 3 , where the training dataset is defined by $x_{1}, \ldots, x_{k} \in \mathcal{D}$ and $i, j \in[1, k]$ ). The equation (3) define the decision function $f(x)$ :

$$
f(x)=\operatorname{sgn}((W . \Psi(x))-\rho),
$$

where weight vector is denoted by $W$, an offset variables $\rho$ is used to create the hyperplane $\mathcal{H}$, with $\Psi$ is the feature map $\mathcal{D} \rightarrow \mathcal{F}$. However, computing $W$ and $\rho$ values requires the resolution of quadratic minimization problem:

$$
\begin{gathered}
\min _{w \in \mathcal{F}, \xi \in R^{l}, \rho \in R} \frac{1}{2}\|w\|^{2} \frac{1}{\nu l} \sum_{i}^{l} \xi_{i}-\rho, \\
\text { subject to }(w . \Psi(x)) \geq \rho-\xi_{i}, \xi_{i} \geq 0
\end{gathered}
$$

The solution is parameterized by $\nu \in[0,1]$.

\section{DBN-OCSVM ALGORITHM}

This section briefly describes the proposed anomaly detection approach that will be used to monitor ozone measurements. This approach combines the advantages of a DBN model and those of OCSVM to enhance ozone pollution monitoring. DBNs have a good ability to discover nonlinearity and learn from high-dimensional data [21].It should be pointed out that DBN modeling do not require assumptions on the distribution of data. In addition, it is a powerful tool to handle the non-linearity in the data. In this study, firstly, a reference 


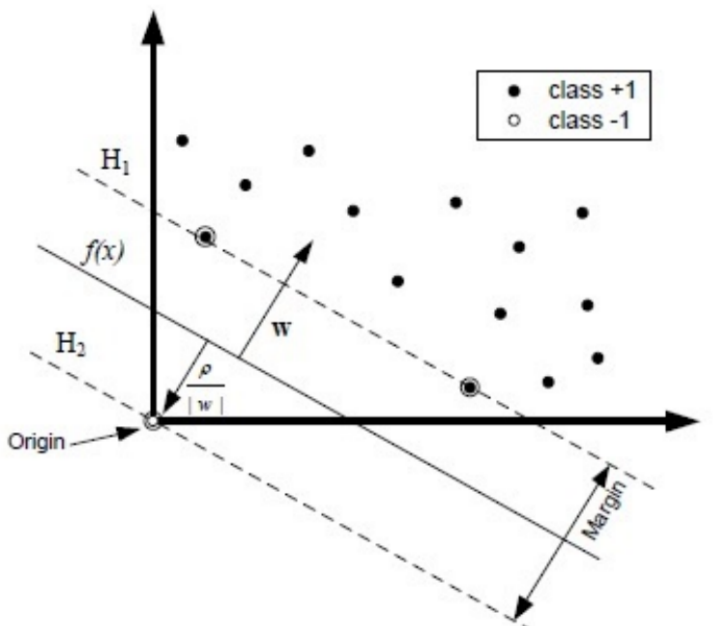

Fig. 3. OCSVM procedure.

BN model consisting of four layers is built using anomalyfree data and then is utilized to predict a new data. Here, to train RBMs, the contrastive divergence learning procedure was used [22]. The DBN model is first built with unsupervised learning of anomaly-free data. The second phase consists in building OCSVM based on the features extracted by DBN to separate normal from abnormal features by building a hyperplane. The steps of the DBN-OCSVM scheme are presented in Algorithm 4.
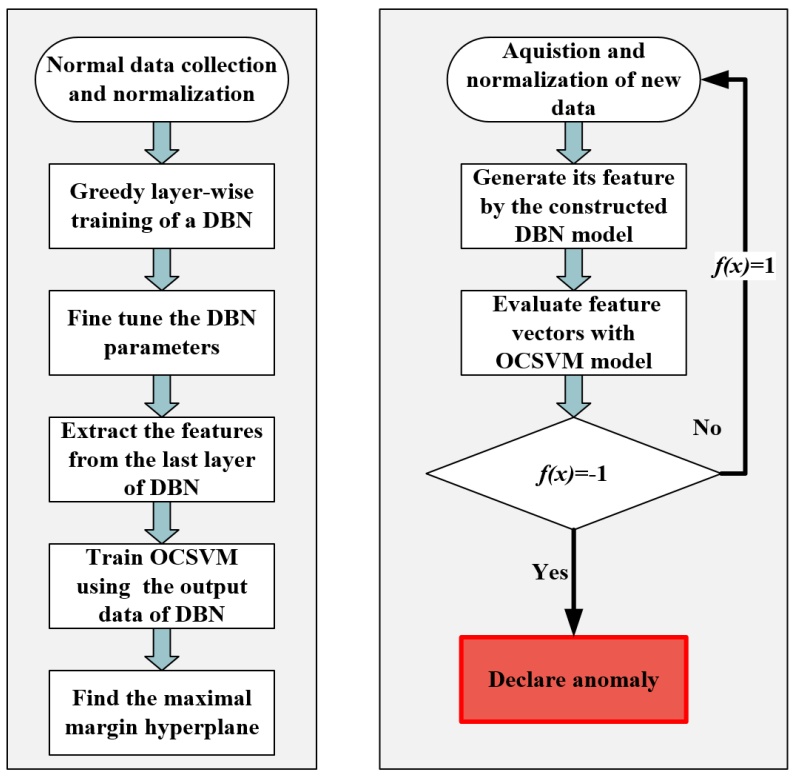

Fig. 4. A diagrammatic representation of the proposed procedure.

\section{OZONE POLLUTION MONITORING}

Ozone measurements from Isère region in France will be monitored in this study. Isère is a department in the AuvergneRhône-Alpes region. It is located in eastern France. Atmo Auvergne-Rhône-Alpes is the organization supervising air quality in Isère region. In this region, there are 14 stations collecting the hourly ozone concentration data. Among them, there are 8 urban stations, 5 peri-urban stations, and one rural station. Each air quality station is a room containing several analyzers. Each analyzer is dedicated to a type of pollutants, such as ozone and PM2.5.

Hourly ozone measurements are used in this work. The time series data of ozone measurements from 1st January to 4th March 2015 (see Figure 5) were used to develop a DBN model.

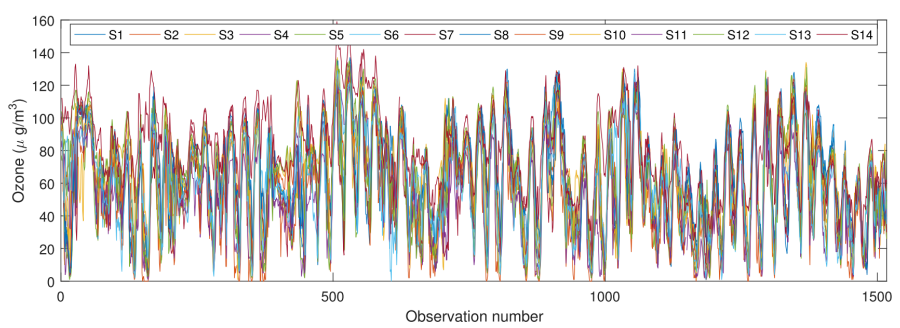

Fig. 5. Training data time series.

Descriptive characteristics of the training ozone time-series data are visually summarized via box plots (e.g., minimum, median, the 25th and 75th percentiles, and maximum) ad shown in Figure 6. Box plot for each time series facilitates the visual comparison of the shape of the corresponding distributions. In Figure 6, each box visually represents the ozone data from the specified station. Generally speaking, if a box has whiskers with approximately equal lengths and the two side of the box are approximately equal, the data has a symmetric distribution. Otherwise, the distribution is skewed. It can be seen from Figure 6 that distributions of data from all stations are approximatively symmetric and have similar behavior.

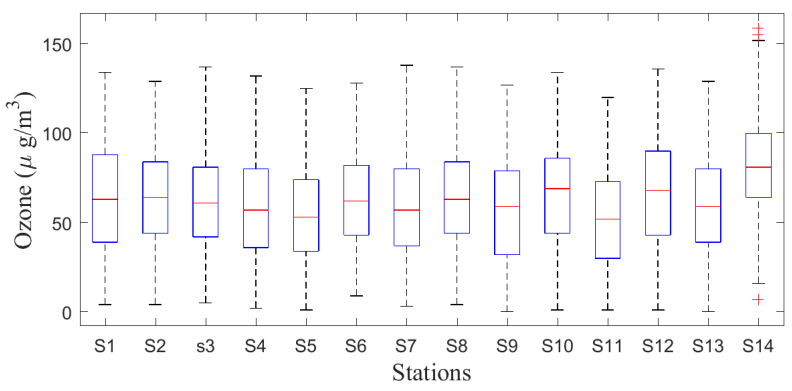

Fig. 6. Boxplots of training data time series.

The training ozone data is used to train the DBN model and OCSVM detector. The experimental parameters of the DBN model and OCSVM algorithm are presented in Table I. Here, we used DBN to model the complex features of ozone data and employs OCSVM to differenciate between normal and abnormal features by building a hyperplane. Both DBN and OCSVM has been constructed entirely in an unsupervised manner.

The testing data covers the period from 1 January 2017 to 10 September 2017. Atmo Auvergne-Rhône-Alpes monitor this air quality monitoring network and informs of vigilance air pollution in case of abnormal pollutions. The objective is to alert the population, but also the prefectural authorities and the communities, of the risk incurred. Table II summarize the detection results of the proposed technique when applied to the testing data. 


\begin{tabular}{l|ll} 
Models & Parameter & Value \\
\hline \multirow{3}{*}{ DBN } & Layers & 3 \\
& Units & 17 \\
& Learning rate & 0.001 \\
& Epochs & 1000 \\
\hline
\end{tabular}

TABLE I

PARAMETERS UTILIZED IN DBN MODEL.

\begin{tabular}{|l|l|l|l|l|l|}
\hline Day & Month & Year & Alert & Period & DBN-OCSVM \\
\hline 29 & August & 2017 & Pollution vigilance & $(13 \mathrm{~h}-20 \mathrm{~h})$ & yes \\
\hline 4 & August & 2017 & Pollution vigilance & $(13 \mathrm{~h}-20 \mathrm{~h})$ & yes \\
\hline 3 & August & 2017 & Pollution vigilance & $(13 \mathrm{~h}-20 \mathrm{~h})$ & yes \\
\hline 7 & July & 2017 & Pollution vigilance & $(13 \mathrm{~h}-20 \mathrm{~h})$ & yes \\
\hline 23 & June & 2017 & Pollution vigilance & $(13 \mathrm{~h}-20 \mathrm{~h})$ & yes \\
\hline 22 & June & 2017 & Pollution vigilance & $(13 \mathrm{~h}-20 \mathrm{~h})$ & yes \\
\hline 20 & June & 2017 & Pollution vigilance & $(13 \mathrm{~h}-20 \mathrm{~h})$ & yes \\
\hline
\end{tabular}

TABLE II

MONITORING RESULTS OF DBN-OCSVM.

Figure 7 shows ozone measurements and their corresponding DBN-OCSVM outputs in the presence of abnormal ozone measurement occurred on 22 and 23 June 2017. We can see that the DBN-based scheme detect all abnormal ozone measurements declared by the experts declared by Atmo Auvergne-Rhône-Alpes air monitoring association.
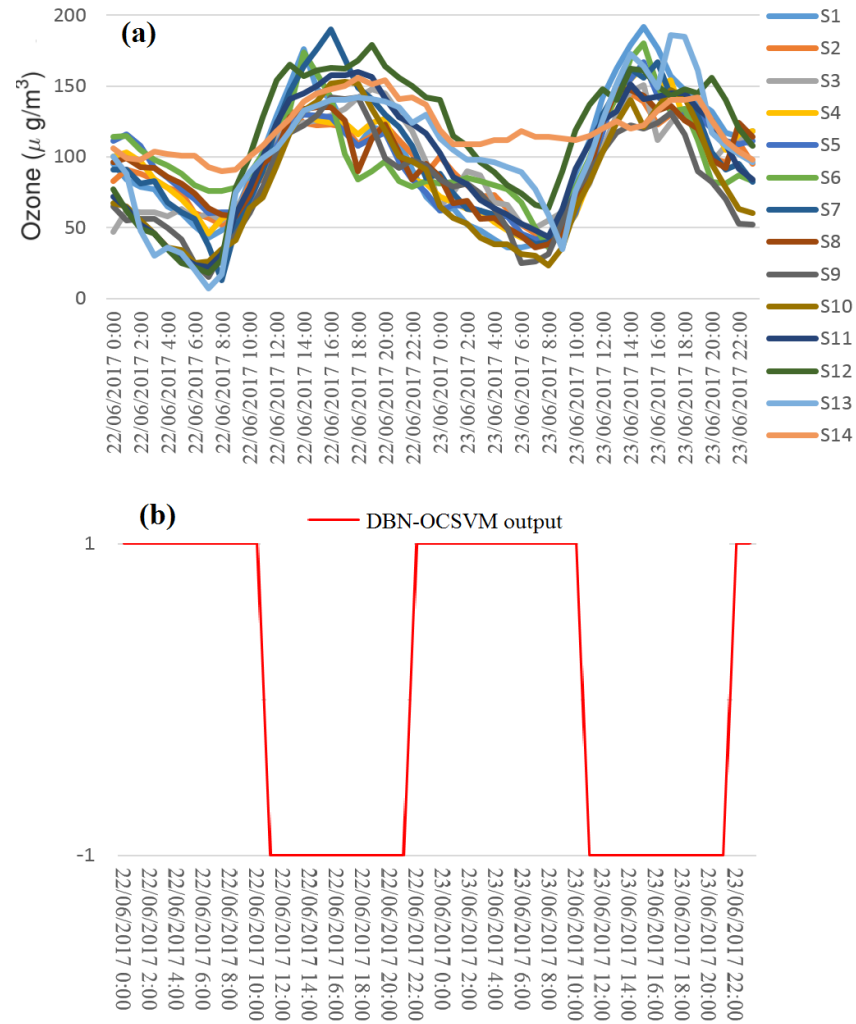

Fig. 7. (a) Ozone time series data. (b) Monitoring results of DBN-OCSVM in the presence of abnormal ozone measurement occurred on 22 and 23 June 2017. The DBN-OCSVM scheme returns a value of ' 1 ' in the presence of abnormal ozone measurements and returns a value of ' -1 ' in the absence of anomalies.

\section{CONCLUSION}

We propose an effective ozone measurements monitoring method that uses ozone measurements data collected from an air quality monitoring network. In this approach, DBN model is employed to capture features from ozone data and OCSVM algorithm is used to detect abnormal ozone measurement. Using real data from a network of air quality monitoring systems in Isére, France, we demonstrate the effectiveness of DBN-OCSVM to detect abnormal ozone pollution. The proposed monitoring strategy was successfully applied to these data. We compared monitoring results of DBN-OCSVM with the results provided by Atmo Auvergne-Rhône-Alpes air monitoring association. Results demonstrate the efficiency of the proposed methodology to identify abnormalities in ozone measurements.

In a network of ozone measurement stations, it is helpful to know where the anomaly has occurred. If a station or a measurement sensor is not functioning well, then, it is essential to know where to intervene in maintenance. In addition, in the case of abnormal ozone pollution, the location of the pollution should be identified. In future, we plan to study the problem of the detection and localization of anomalies in ozone measurements.

\section{ACKNOWLEDGEMENT}

The research reported in this publication was supported by funding from King Abdullah University of Science and Technology (KAUST) Office of Sponsored Research (OSR) under Award No: OSR-2015-CRG4-2582.

\section{REFERENCES}

[1] K. B. Shaban, A. Kadri, and E. Rezk, "Urban air pollution monitoring system with forecasting models," IEEE Sensors Journal, vol. 16, no. 8 , pp. 2598-2606, 2016.

[2] M. P. Rissanen, T. Kurtén, M. Sipilal, J. A. Thornton, J. Kangasluoma, N. Sarnela, H. Junninen, S. Jørgensen, S. Schallhart, M. K. Kajos et al., "The formation of highly oxidized multifunctional products in the ozonolysis of cyclohexene," Journal of the American Chemical Society, vol. 136, no. 44, pp. 15 596-15 606, 2014.

[3] A. Nawahda, "An assessment of adding value of traffic information and other attributes as part of its classifiers in a data mining tool set for predicting surface ozone levels," Process Safety and Environmental Protection, vol. 99, pp. 149-158, 2016.

[4] C. Vlachokostas, S. Nastis, C. Achillas, K. Kalogeropoulos, I. Karmiris, N. Moussiopoulos, E. Chourdakis, G. Banias, and N. Limperi, "Economic damages of ozone air pollution to crops using combined air quality and GIS modelling," Atmospheric Environment, vol. 44, no. 28, pp. 3352-3361, 2010.

[5] C. Dueñas, M. Fernández, S. Canete, J. Carretero, and E. Liger, "Analyses of ozone in urban and rural sites in málaga (spain)," Chemosphere, vol. 56, no. 6, pp. 631-639, 2004.

[6] F. Harrou, F. Kadri, S. Khadraoui, and Y. Sun, "Ozone measurements monitoring using data-based approach," Process Safety and Environmental Protection, vol. 100, pp. 220-231, 2016.

[7] F. Harrou, L. Fillatre, M. Bobbia, and I. Nikiforov, "Statistical detection of abnormal ozone measurements based on constrained generalized likelihood ratio test," in Decision and Control (CDC), 2013 IEEE 52nd Annual Conference on. IEEE, 2013, pp. 4997-5002.

[8] F. Harrou, M. Nounou, and H. Nounou, "Statistical detection of abnormal ozone levels using principal component analysis," ternational Journal of Engineering \& Technology, vol. 12, no. 6, pp. 54-59, 2012. 
[9] Y. Jia, J. Wu, and Y. Du, "Traffic speed prediction using deep learning method," in Intelligent Transportation Systems (ITSC), 2016 IEEE 19th International Conference on. IEEE, 2016, pp. 1217-1222.

[10] D. Ravì, C. Wong, F. Deligianni, M. Berthelot, J. Andreu-Perez, B. Lo, and G.-Z. Yang, "Deep learning for health informatics," IEEE journal of biomedical and health informatics, vol. 21, no. 1, pp. 4-21, 2017.

[11] O. Costilla-Reyes, P. Scully, and K. B. Ozanyan, "Deep neural networks for learning spatio-temporal features from tomography sensors," IEEE Transactions on Industrial Electronics, 2017.

[12] A. Koesdwiady, R. Soua, and F. Karray, "Improving traffic flow prediction with weather information in connected cars: A deep learning approach," IEEE Transactions on Vehicular Technology, vol. 65, no. 12, pp. 9508-9517, 2016.

[13] A. Dairi, F. Harrou, M. Senouci, and Y. Sun, "Unsupervised obstacle detection in driving environments using deep-learning-based stereovision," Robotics and Autonomous Systems, vol. 100, pp. 287-301, 2018.

[14] F. Harrou, A. Dairi, Y. Sun, and M. Senouci, "Wastewater treatment plant monitoring via a deep learning approach," in 2018 IEEE International Conference on Industrial Technology (ICIT). IEEE, 2018, pp. 15441548.

[15] J. Wang, X. Zhang, Q. Gao, H. Yue, and H. Wang, "Device-free wireless localization and activity recognition: A deep learning approach," IEEE Transactions on Vehicular Technology, vol. 66, no. 7, pp. 6258-6267, 2017.

[16] X. Wang, L. Gao, S. Mao, and S. Pandey, "CSI-based fingerprinting for indoor localization: A deep learning approach," IEEE Transactions on Vehicular Technology, vol. 66, no. 1, pp. 763-776, 2017.

[17] G. Hinton, "A practical guide to training restricted boltzmann machines," in Neural networks: Tricks of the trade. Springer, 2012, pp. 599-619.

[18] G. E. Hinton and T. J. Sejnowski, "Learning and releaming in boltzmann machines," Parallel distributed processing: Explorations in the microstructure of cognition, vol. 1, no. 282-317, p. 2, 1986.

[19] G. E. Hinton, S. Osindero, and Y.-W. Teh, "A fast learning algorithm for deep belief nets," Neural computation, vol. 18, no. 7, pp. 1527-1554, 2006.

[20] B. Schölkopf, J. C. Platt, J. Shawe-Taylor, A. J. Smola, and R. C. Williamson, "Estimating the support of a high-dimensional distribution," Neural computation, vol. 13, no. 7, pp. 1443-1471, 2001.

[21] Y. Bengio, P. Lamblin, D. Popovici, and H. Larochelle, "Greedy layerwise training of deep networks," in Advances in neural information processing systems, 2007, pp. 153-160.

[22] G. E. Hinton, "Training products of experts by minimizing contrastive divergence," Neural computation, vol. 14, no. 8, pp. 1771-1800, 2002. 